\title{
The perimeter and the site-perimeter of set partitions
}

\author{
Toufik Mansour \\ Department of Mathematics \\ University of Haifa \\ 3498838 Haifa, Israel \\ tmansour@univ.haifa.ac.il
}

Submitted: Oct 18, 2018; Accepted: Apr 30, 2019; Published: May 31, 2019

(C) The author. Released under the CC BY-ND license (International 4.0).

\begin{abstract}
In this paper, we study the generating function for the number of set partitions of $[n]$ represented as bargraphs according to the perimeter/site-perimeter. In particular, we find explicit formulas for the total perimeter and the total site-perimeter over all set partitions of $[n]$.
\end{abstract}

Mathematics Subject Classifications: 05A18

\section{Introduction}

A partition of a positive integer $n$ is a non-increasing sequence of positive integers, called parts, whose sum is $n$. A composition is a partition in which the parts may come in any order, as originally defined by MacMahon [11]. Compositions can be represented as bargraphs. A bargraph is a column convex polyomino such that the lower edge lies on a horizontal axis, when it is drawn on a regular planar lattice grid and is made up of square cells. Clearly, the number parts and the size of a composition is the number columns and the total number of cells in the representing bargraph, respectively. For instance, Figure 1 presents the bargraph 122341411. Recently, statistics on bargraphs have been

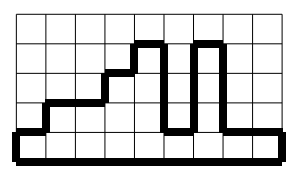

Figure 1: The bargraph 122341411

received a lot of attention. For instance, in $[10,17]$ it is found the generating function for 
the number of bargraphs according to the number of horizontal and up steps. In [14] it is studied the generating function for the number of bargraphs according to the number of interior vertices and edges. Moreover, Blecher et al. counted bargraphs according to statistics: descents [3], levels [1], peaks [2] and walls [4].

The enumeration of polyominoes according to their area and perimeter is an interesting problem in combinatorics [7,9]. When one studies combinatorial families presented geometrically, the perimeter and the area are the most natural and most important statistics to be considered. The perimeter of a bargraph $B$, denoted by $\operatorname{per}(B)$, is the number of edges on the boundary of $B$. The site-perimeter of a bargraph $B$, denoted by $\operatorname{sper}(B)$, is the number of nearest-neighbor cells outside the boundary of $B$. The perimeter and the site perimeter of words were studied in [5] and [6], respectively. Motivated by these results, we extend the study of perimeter/site-perimeter to set partitions.

A set partition of $[n]$ is any collection of nonempty, pairwise disjoint subsets, called blocks, whose union is $[n]$ (there is a single empty set partition of [0] which has no blocks). We denote the set of all set partitions of $[n]$ by $P(n)$ and the set of all set partitions of $[n]$ with $k$ blocks by $P(n, k)$. We will write a set partition $\pi \in P(n, k)$ as $\pi=$ $B_{1} / B_{2} / \cdots / B_{k}$, where $\min \left(B_{1}\right)<\min \left(B_{2}\right)<\cdots<\min \left(B_{k}\right)$. Equivalently, we will write a set partition by the canonical sequential form $\pi=\pi_{1} \pi_{2} \cdots \pi_{n}$, wherein $i \in B_{\pi_{i}}$ for $i \in[n]$ (see, e.g., [12, 19]). For example, the set partition $\pi=\{1,4,8,9\} /\{2,3\} /\{5\} /\{6,7\}$ has the canonical sequential form $\pi=122134411$. We will represent each set partition $\pi$ by the corresponding bargraph of the canonical sequential form of $\pi$. For instance, Figure 1 represents the bargraph of $\pi=\{1,6,8,9\} /\{2,3\} /\{4\} /\{5,7\}$. Recently, statistics on bargraphs of set partitions have been investigated by several authors (see, e.g., [13, 15, $16])$.

The aim of this paper is to study the perimeter (see Section 2) and the site-perimeter (see Section 3) of set partitions. For example, the perimeter and the site-perimeter of the set partition 122341411 are 32 and 24, respectively. In particular, we show that the total of the half of the perimeter over all set partitions of $[n]$ is given by (see Corollary 5 )

$$
a_{n}=\frac{n+3}{6} B_{n+1}-\frac{5}{36} B_{n+2}+\frac{18 n+13}{18} B_{n}-\frac{6 n+7}{36} B_{n-1}
$$

and the total of the site-perimeter over all set partitions of $[n]$ is given by (see Corollary 7)

$$
\begin{aligned}
b_{n} & =\left(\frac{n}{4}+\frac{73}{72}\right) B_{n+1}-\frac{11}{48} B_{n+2}+\left(\frac{4 n}{3}+\frac{91}{48}\right) B_{n}+\left(\frac{n}{4}-\frac{67}{72}\right) B_{n-1} \\
& +\left(\frac{n}{6}-\frac{23}{144}\right) B_{n-2},
\end{aligned}
$$

where $B_{n}$ is the $n$th Bell number.

In order to obtain asymptotic estimates for the moments as well as the limiting distribution, we need

$$
B_{n+h}=B_{n} \frac{(n+h) !}{n ! r^{h}}\left(1+O\left(\frac{\log n}{n}\right)\right)
$$


uniformly for $h=O(\log n)$, where $B_{n}$ is the $n$th Bell number and $r$ is the positive root of $r e^{r}=n+1$. See [8] for an even stronger form that includes further terms in the asymptotic expansion. We say that the sequence $f_{n}$ is asymptotically equivalent to the sequence $g_{n}$, denoted by $f_{n} \sim g_{n}$, if $\lim _{n \rightarrow \infty} \frac{f_{n}}{g_{n}}=1$. So,

$$
\begin{aligned}
\frac{a_{n}}{B_{n}} & \sim \frac{n+3}{6} \frac{B_{n+1}}{B_{n}}-\frac{5}{36} \frac{B_{n+2}}{B_{n}}+\frac{18 n+13}{18}-\frac{6 n+7}{36} \frac{B_{n-1}}{B_{n}} \\
& \sim \frac{n+3}{6} \frac{B_{n+1}}{B_{n}}-\frac{5}{36} \frac{B_{n+2}}{B_{n}}
\end{aligned}
$$

and

$$
\begin{aligned}
\frac{b_{n}}{B_{n}} & \sim \frac{36 n+146}{144} \frac{B_{n+1}}{B_{n}}-\frac{33}{144} \frac{B_{n+2}}{B_{n}}+\frac{192 n+273}{144}-\frac{36 n-134}{144} \frac{B_{n-1}}{B_{n}}+\frac{24 n-23}{144} \frac{B_{n-2}}{B_{n}} \\
& \sim \frac{36 n+146}{144} \frac{B_{n+1}}{B_{n}}-\frac{33}{144} \frac{B_{n+2}}{B_{n}},
\end{aligned}
$$

which, by (1), leads to the following corollary.

Corollary 1. Asymptotically,

$$
a_{n}=\frac{n^{2} B_{n}}{6(\log (n)-\log \log n)}\left(1-\frac{5}{6(\log n-\log \log n)}\right)\left(1+O\left(\frac{\log n}{n}\right)\right)
$$

and

$$
b_{n}=\frac{n^{2} B_{n}}{48(\log (n)-\log \log n)}\left(12-\frac{11}{\log n-\log \log n}\right)\left(1+O\left(\frac{\log n}{n}\right)\right) .
$$

\section{The perimeter of set partitions}

Let $P_{k}(x, q)=\sum_{n \geqslant k} \sum_{\pi \in P_{n, k}} x^{n} q^{\frac{1}{2} p e r(\pi)}$ be the generating function for the number of set partitions of $n$ with exactly $k$ blocks, according to the half of the perimeter. Generally, let $P_{k}\left(x, q \mid a_{1} a_{2} \cdots a_{s}\right)=\sum_{n \geqslant k} \sum_{\pi=\pi^{\prime} a_{1} a_{2} \cdots a_{s} \in P_{n, k}} x^{n} q^{\frac{1}{2} \operatorname{per}(\pi)}$ be the generating function for the number of set partitions $\pi=\pi^{\prime} a_{1} a_{2} \cdots a_{s}$ of $n$ with exactly $k$ blocks, according to the half of the perimeter. We define $P_{0}(x, q)=1$. Since each set partition with one block has the form $11 \cdots 1$, we have $P_{1}(x, q)=\frac{x q^{2}}{1-x q}$. By definitions we have

$$
P_{k}(x, q \mid a)=\sum_{b=1}^{k} P_{k}(x, q \mid b a)=x \sum_{b=1}^{a} q^{a-b+1} P_{k}(x, q \mid b)+x q \sum_{b=a+1}^{k} P_{k}(x, q \mid b),
$$

for all $1 \leqslant a \leqslant k-1$. Moreover,

$$
P_{k}(x, q \mid k)=x \sum_{b=1}^{k} q^{k-b+1} P_{k}(x, q \mid b)+x \sum_{b=1}^{k-1} q^{k-b+1} P_{k-1}(x, q \mid b) .
$$


Define $P_{k}(x, q, v)=\sum_{a=1}^{k} P_{k}(x, q \mid a) v^{a-1}$. Then (2)-(3) can be written as

$$
\begin{aligned}
P_{k}(x, q, v) & =P_{k}(x, q \mid k) v^{k-1}+\frac{x}{1-q v}\left(q P_{k}(x, q, v)-q^{k} v^{k-1} P_{k}(x, q, 1 / q)\right) \\
& +\frac{x q}{1-v}\left(P_{k}(x, q, 1)-P_{k}(x, q, v)\right)
\end{aligned}
$$

with

$$
P_{k}(x, q \mid k)=x q^{k} P_{k}(x, q, 1 / q)+x q^{k} P_{k-1}(x, q, 1 / q) .
$$

Thus, we can state the following result.

Proposition 2. We have

$$
\begin{aligned}
P_{k}(x, q, v) & =x q^{k} v^{k-1} P_{k-1}(x, q, 1 / q)+\frac{x q}{1-q v}\left(P_{k}(x, q, v)-q^{k} v^{k} P_{k}(x, q, 1 / q)\right) \\
& +\frac{x q}{1-v}\left(P_{k}(x, q, 1)-P_{k}(x, q, v)\right)
\end{aligned}
$$

with $P_{1}(x, q, v)=\frac{x q^{2}}{1-x q}$.

Proposition 2 with $q=1$ gives $P_{k}(x, 1, v)=x v^{k-1} P_{k-1}(x, 1,1)+\frac{x\left(1-v^{k}\right)}{1-v} P_{k}(x, 1,1)$ with $P_{1}(x, 1, v)=\frac{x}{1-x}$. If we take $v \rightarrow 1$, then we obtain $P_{k}(x, 1,1)=\frac{x}{1-k x} P_{k-1}(x, 1,1)$ with $P_{1}(x, 1,1)=\frac{x}{1-x}$, which leads to $P_{k}(x, 1,1)=\frac{x^{k}}{(1-x) \cdots(1-k x)}$, as expected. Thus, for all $k \geqslant 1$,

$$
P_{k}(x, 1, v)=\frac{x^{k}\left(v^{k-1}(1-v)(1-k x)+x\left(1-v^{k}\right)\right)}{(1-v)(1-x) \cdots(1-k x)} .
$$
have

Define $F_{k}(x, v)=\left.\frac{\partial}{\partial q} P_{k}(x, q, v)\right|_{q=1}$ and $G_{k}(x, v)=\frac{\partial}{\partial v} P_{k}(x, 1, v)$. By Proposition 2, we

$$
\begin{aligned}
F_{k}(x, v) & =k x v^{k-1} P_{k-1}(x, 1,1)+x v^{k-1} F_{k-1}(x, 1)-x v^{k-1} G_{k-1}(x, 1) \\
& +\frac{x}{(1-v)^{2}}\left(P_{k}(x, 1, v)-v^{k} P_{k}(x, 1,1)\right) \\
& +\frac{x}{1-v}\left(F_{k}(x, v)-k v^{k} P_{k}(x, 1,1)-v^{k} F_{k}(x, 1)+v^{k} G_{k}(x, 1)\right) \\
& +\frac{x}{1-v}\left(P_{k}(x, 1,1)-P_{k}(x, 1, v)\right)+\frac{x}{1-v}\left(F_{k}(x, 1)-F_{k}(x, v)\right)
\end{aligned}
$$

with $F_{1}(x, v)=\frac{x(2-x)}{(1-x)^{2}}$. Thus, by using (4) and taking $v \rightarrow 1$, we obtain

$$
F_{k}(x, 1)=\frac{x}{1-k x} F_{k-1}(x, 1)+\frac{x^{k}\left(12+3\left(k^{2}-5 k+2\right) x-k(2 k-7)(k-1) x^{2}\right)}{6(1-k x) \prod_{j=1}^{k}(1-j x)}
$$

with $F_{0}(x, 1)=0$. Thus by induction, we can state the following result. 
Theorem 3. The generating function $F_{k}(x, 1)$ for the total of the half of the perimeter over all set partitions of $[n]$ with exactly $k$ blocks is given by

$$
\frac{x^{k}}{6 \prod_{j=1}^{k}(1-j x)} \sum_{i=1}^{k} \frac{12+3\left(i^{2}-5 i+2\right) x-i(i-1)(2 i-7) x^{2}}{1-i x} .
$$

In order to study further the total of the half of the perimeter over all set partitions of $[n]$, we consider the exponential generating function $E_{k}(x)=\sum_{n \geqslant 0}\left[x^{n}\right]\left(F_{k}(x, 1)\right) \frac{x^{n}}{n !}$, where $\left[x^{n}\right] f(x)$ denotes the coefficient of $x^{n}$ in the generating function $f(x)$. Note that $\frac{x^{k}}{\prod_{j=1}^{k}(1-j x)}=\sum_{n \geqslant 0} S_{n, k} x^{n}$ and $\frac{1}{k !}\left(e^{x}-1\right)^{k}=\sum_{n \geqslant 0} S_{n, k} \frac{x^{n}}{n !}$, where $S_{n, k}$ denotes the Stirling number of the second kind (for example, see [12]). So, by (5), we have

$$
(1-k x) F_{k}(x, 1)=x F_{k-1}(x, 1)+\frac{12+3\left(k^{2}-5 k+2\right) x-k(2 k-7)(k-1) x^{2}}{6} \sum_{n \geqslant 0} S_{n, k} x^{n}
$$

with $F_{0}(x, 1)=0$. Thus, the exponential generating function $E_{k}(x)$ satisfies,

$$
\begin{aligned}
E_{k}(x) & =k \int_{0}^{x} E_{k}(t) d t+\int_{0}^{x} E_{k-1}(t) d t+\frac{2\left(e^{x}-1\right)^{k}}{k !} \\
& +\frac{k^{2}-5 k+2}{2} \int_{0}^{x} \frac{\left(e^{t}-1\right)^{k}}{k !} d t-\frac{k(2 k-7)(k-1)}{6} \int_{0}^{x} \int_{0}^{t} \frac{\left(e^{r}-1\right)^{k}}{k !} d r d t
\end{aligned}
$$

with $E_{0}(x)=0$. Hence,

$$
\begin{aligned}
\frac{d^{2}}{d x^{2}} E_{k}(x) & =k \frac{d}{d x} E_{k}(x)+\frac{d}{d x} E_{k-1}(x)+\frac{2 e^{x}\left(e^{x}-1\right)^{k-2}\left(k e^{x}-1\right)}{(k-1) !} \\
& +\frac{k^{2}-5 k+2}{2} \frac{\left(e^{x}-1\right)^{k-1} e^{x}}{(k-1) !}-\frac{k(2 k-7)(k-1)}{6} \frac{\left(e^{x}-1\right)^{k}}{k !}
\end{aligned}
$$

Define $E(x, y)=\sum_{k \geqslant 0} E_{k}(x) y^{k}$. By multiplying by $y^{k}$, and summing over $k \geqslant 1$, we obtain

$$
\begin{aligned}
\frac{\partial^{2}}{\partial x^{2}} E(x, y) & =y \frac{\partial^{2}}{\partial x \partial y} E(x, y)+y \frac{\partial}{\partial x} E(x, y) \\
& +\frac{1}{6} y\left(y^{2} e^{3 x}+9 y e^{2 x}+3\left(2-y^{2}\right) e^{x}+2 y^{2}+3 y\right) e^{y\left(e^{x}-1\right)}
\end{aligned}
$$

Solving this partial differential equation under the condition $\left.\frac{\partial}{\partial x} E(x, y)\right|_{x=0}=2 y$, we obtain the following result.

Theorem 4. The exponential generating function $E(x, y)$ for the total of the half of the perimeter over all set partitions of $[n]$ with exactly $k$ blocks is given by

$$
\frac{y}{36} \int_{0}^{x}\left((6 t-5) y^{2} e^{3 t}+9(6 t+1) y e^{2 t}+9\left(y^{2}+4 t+8\right) e^{t}-y(4 y+9)\right) e^{y e^{t}-y} d t
$$


By Theorem 4 we have

$$
\begin{aligned}
& \frac{\partial}{\partial x} E(x, y) \\
& =\frac{y}{36}\left((6 x-5) y^{2} e^{3 x}+9(6 x+1) y e^{2 x}+9\left(y^{2}+4 x+8\right) e^{x}-y(4 y+9)\right) e^{y e^{x}-y} \\
& =\frac{6 x-5}{36} \frac{\partial^{3}}{\partial x^{3}} e^{y e^{x}-y}+\frac{3 x+2}{3} \frac{\partial^{2}}{\partial x^{2}} e^{y e^{x}-y}+\frac{9 y^{2}-6 x+53}{36} \frac{\partial}{\partial x} e^{y e^{x}-y}-\frac{4 y^{3}+9 y^{2}}{36} e^{y e^{x}-y} .
\end{aligned}
$$

By comparing the coefficient of $x^{n} y^{k} / n$ ! in both sides, we get the following formula.

Corollary 5. The total of the half of the perimeter over all set partitions of $[n+1]$ with exactly $k$ blocks is given by

$$
\begin{aligned}
\frac{n+4}{6} S_{n+2, k} & -\frac{5}{36} S_{n+3, k}+\frac{36 n+53}{36} S_{n+1, k} \\
& +\frac{1}{4} S_{n+1, k-2}-\frac{n}{6} S_{n, k}-\frac{1}{9} S_{n, k-3}-\frac{1}{4} S_{n, k-2},
\end{aligned}
$$

and the total of the half of the perimeter over all set partitions of $[n+1]$ is given by

$$
\frac{n+4}{6} B_{n+2}-\frac{5}{36} B_{n+3}+\frac{18 n+31}{18} B_{n+1}-\frac{6 n+13}{36} B_{n},
$$

where $S_{n, k}$ denotes the Stirling number of the second kind and $B_{n}$ denotes the nth Bell number.

\section{The site-perimeter of set partitions}

Let $Q_{k}(x, q)=\sum_{n \geqslant k} \sum_{\pi \in P_{n, k}} x^{n} q^{s p e r(\pi)}$ be the generating function for the number of set partitions of $n$ with $k$ blocks according to the site-perimeter. Generally, let

$$
Q_{k}\left(x, q \mid a_{1} a_{2} \cdots a_{s}\right)=\sum_{n \geqslant k} \sum_{\pi=\pi^{\prime} a_{1} a_{2} \cdots a_{s} \in P_{n, k}} x^{n} q^{\operatorname{sper}(\pi)}
$$

be the generating function for the number of set partition $\pi=\pi^{\prime} a_{1} a_{2} \cdots a_{s}$ of $n$ with $k$ blocks according to the site-perimeter. We define $Q_{0}(x, q)=1$. Since each set partition with one block has the form $11 \cdots 1$, we have

$$
Q_{1}(x, q)=\frac{x q^{4}}{1-x q^{2}}
$$

Since each set partition with two blocks has either the form $11 \cdots 12 \pi^{\prime} 2$ or the form $11 \cdots 12 \pi^{\prime} 1$, where $\pi^{\prime}$ is a word over alphabet $\{1,2\}$, we obtain

$$
Q_{2}(x)=\frac{q^{7} x^{2}}{1-2 q^{2} x}+\left(\frac{q^{6} x^{2}}{1-2 q^{2} x}-\frac{q^{6} x^{2}}{1-q^{2} x}\right)=\frac{q^{7} x^{2}\left(1+q x-q^{2} x\right)}{\left(1-2 q^{2} x\right)\left(1-q^{2} x\right)} .
$$


Clearly, $Q_{k}(x, q)=\sum_{a=1}^{k} Q_{k}(x, q \mid a)$ and $Q_{k}(x, q \mid a)=\sum_{b=1}^{k} Q_{k}(x, q \mid b a)$ for all $1 \leqslant a \leqslant$ $k-1$. By the definitions we have

$$
\begin{aligned}
Q_{k}(x, q \mid a) & =\sum_{b=1}^{a-1} Q_{k}(x, q \mid b a)+x q^{2} Q_{k}(x, q \mid a)+x q \sum_{b=a+1}^{k} Q_{k}(x, q \mid b), \\
Q_{k}(x, q \mid b a) & =x q^{2 a-2 b+1} \sum_{c=1}^{b} Q_{k}(x, q \mid c b)+x \sum_{c=b+1}^{a} q^{2 a-b-c+2} Q_{k}(x, q \mid c b) \\
& +x \sum_{c=a+1}^{k} q^{a-b+2} Q_{k}(x, q \mid c b),
\end{aligned}
$$

where $1 \leqslant b \leqslant a-1$ and $1 \leqslant a \leqslant k-1$. Moreover,

$$
\begin{aligned}
Q_{k}(x, q \mid k) & =\sum_{b=1}^{k-1} Q_{k}(x, q \mid b k)+x q^{2} Q_{k}(x, q \mid k), \\
Q_{k}(x, q \mid b k) & =x q^{2 k-2 b+1} \sum_{c=1}^{b}\left(Q_{k}(x, q \mid c b)+Q_{k-1}(x, q \mid c b)\right) \\
& +x \sum_{c=b+1}^{k-1} q^{2 k-b-c+2}\left(Q_{k}(x, q \mid c b)+Q_{k-1}(x, q \mid c b)\right)+x q^{k-b+2} Q_{k}(x, q \mid k b),
\end{aligned}
$$

where $1 \leqslant b \leqslant k-1$.

Our goal is to find a recurrence relation for the generating function

$$
Q_{k}^{\prime}(x)=\left.\frac{\partial}{\partial q} Q_{k}(x, q)\right|_{q=1} .
$$

To do that, let $H_{k}(x)=\frac{x^{k}}{\prod_{j=1}^{k}(1-j x)}$ to be the generating function for the number of set partitions of $n$ with exactly $k$ blocks (see [12]). Clearly,

$$
\begin{aligned}
& Q_{k}(x, 1 \mid a)=x H_{k}(x), \quad a=1,2, \ldots, k-1, \\
& Q_{k}(x, 1 \mid k)=x H_{k-1}(x)+x H_{k}(x) .
\end{aligned}
$$

Define $Q_{k}^{\prime}(x \mid a)=\left.\frac{\partial}{\partial q} Q_{k}(x, q \mid a)\right|_{q=1}$ and $Q_{k}^{\prime}(x \mid b a)=\left.\frac{\partial}{\partial q} Q_{k}(x, q \mid b a)\right|_{q=1}$. Differentiating (6)-(7) at $q=1$ gives

$$
\begin{aligned}
Q_{k}^{\prime}(x \mid a) & =\sum_{b=1}^{a-1} Q_{k}^{\prime}(x \mid b a)+x Q_{k}(x, 1 \mid a)+x \sum_{b=a}^{k} Q_{k}(x, 1 \mid b)+x \sum_{b=a}^{k} Q_{k}^{\prime}(x \mid b), \\
Q_{k}^{\prime}(x \mid b a) & =x(2 a-2 b+1) \sum_{c=1}^{b} Q_{k}(x, 1 \mid c b)+x \sum_{c=b+1}^{a}(2 a-b-c+2) Q_{k}(x, 1 \mid c b) \\
& +x \sum_{c=a+1}^{k}(a-b+2) Q_{k}(x, 1 \mid c b)+x \sum_{c=1}^{k} Q_{k}^{\prime}(x \mid c b),
\end{aligned}
$$


where $1 \leqslant b \leqslant a-1$ and $1 \leqslant a \leqslant k-1$. Thus, by (10)-(11), we have

$$
Q_{k}^{\prime}(x \mid b a)=\frac{x^{2}\left(\left(a^{2}-b^{2}-a-b\right) x+2 a-2 b+4\right)}{2} H_{k}(x)+x Q_{k}^{\prime}(x \mid b),
$$

which, by substituting into $Q_{k}^{\prime}(x \mid a)$ and using (10)-(11), implies

$$
\begin{aligned}
Q_{k}^{\prime}(x \mid a) & =\sum_{b=1}^{a-1} \frac{x^{2}\left(\left(a^{2}-b^{2}-a-b\right) x+2 a-2 b+4\right)}{2} H_{k}(x) \\
& +(k-a+2) x^{2} H_{k}(x)+x^{2} H_{k-1}(x)+x Q_{k}^{\prime}(x),
\end{aligned}
$$

for all $a=1,2, \ldots, k-1$. Therefore, by summing over $a=1,2, \ldots, k-1$ we obtain

$$
\begin{aligned}
Q_{k}^{\prime}(x) & =\frac{1}{12} x(k-1)\left(k(k-2)(k-3) x^{2}+2 k(k+1) x+12\right) H_{k}(x) \\
& +Q_{k}^{\prime}(x \mid k)+(k-1) x Q_{k}^{\prime}(x) .
\end{aligned}
$$

Now, we focus on $Q_{k}^{\prime}(x \mid k)$. By differentiating (8)-(9), we have

$$
\begin{aligned}
Q_{k}^{\prime}(x \mid k) & =\sum_{b=1}^{k-1} Q_{k}^{\prime}(x \mid b k)+2 x Q_{k}(x, 1 \mid k)+x Q_{k}^{\prime}(x \mid k), \\
Q_{k}^{\prime}(x \mid b k) & =x(2 k-2 b+1) \sum_{c=1}^{b}\left(Q_{k}(x, 1 \mid c b)+Q_{k-1}(x, 1 \mid c b)\right) \\
& +x \sum_{c=b+1}^{k-1}(2 k-b-c+2)\left(Q_{k}(x, 1 \mid c b)+Q_{k-1}(x, 1 \mid c b)\right) \\
& +x(k-b+2) Q_{k}(x, 1 \mid k b)+x Q_{k}^{\prime}(x \mid b)+x Q_{k-1}^{\prime}(x \mid b),
\end{aligned}
$$

where $1 \leqslant b \leqslant k-1$. Thus, by substituting expression of $Q_{k}^{\prime}(x \mid b k)$ into expression of $Q_{k}^{\prime}(x \mid k)$ with using (10)-(11), we obtain

$$
\begin{aligned}
Q_{k}^{\prime}(x \mid k) & =\frac{1}{6}\left(2 k(k-2)(k-4) x^{2}+3\left(k^{2}-k-4\right) x+18\right)(1-(k-1) x) H_{k}(x) \\
& +x Q_{k}^{\prime}(x)+x Q_{k-1}^{\prime}(x) .
\end{aligned}
$$

By substituting (13) into (12), we obtain that the generating function $Q_{k}^{\prime}(x)$ satisfies

$$
\begin{aligned}
Q_{k}^{\prime}(x) & =\frac{36+6 k(k-5) x-12\left(k^{2}-4 k+2\right) x^{2}-k(k-1)(k-2)(3 k-13) x^{3}}{12(1-k x)} H_{k}(x) \\
& +\frac{x}{1-k x} Q_{k-1}^{\prime}(x),
\end{aligned}
$$

for all $k \geqslant 3$. Note that $Q_{0}^{\prime}(x)=0, Q_{1}^{\prime}(x)=\frac{2(2-x)}{(1-x)^{2}}$ and $Q_{2}^{\prime}(x)=\frac{x^{2}\left(7-16 x+9 x^{2}-2 x^{3}\right)}{(1-2 x)^{2}(1-x)^{2}}$. By (14), we can introduce first values of the total of the site-perimeter over all set partitions of $[n]$ with $k$ blocks, see Table 1 . 


\begin{tabular}{c||ccccccc}
$k \backslash n$ & 1 & 2 & 3 & 4 & 5 & 6 & 7 \\
\hline \hline 1 & 4 & 6 & 8 & 10 & 12 & 14 & 16 \\
2 & 0 & 7 & 26 & 74 & 188 & 450 & 1040 \\
3 & 0 & 0 & 10 & 71 & 345 & 1426 & 5398 \\
4 & 0 & 0 & 0 & 13 & 151 & 1122 & 6816 \\
5 & 0 & 0 & 0 & 0 & 16 & 276 & 2915 \\
\hline
\end{tabular}

Table 1: The total of the site-perimeter over all set partitions of $[n]$ with $k$ blocks, where $n=1,2, \ldots, 7$ and $k=1,2, \ldots, 5$.

To study further the total of the site-perimeter over all set partitions of $[n]$, we consider the exponential generating function $R_{k}(x)=\sum_{n \geqslant 0}\left[x^{n}\right]\left(Q_{k}^{\prime}(x)\right) \frac{x^{n}}{n !}$. By $(14)$, we have

$$
\begin{aligned}
& (1-k x) Q_{k}^{\prime}(x) \\
& =\left(3+\frac{1}{2} k(k-5) x-\left(k^{2}-4 k+2\right) x^{2}-\frac{1}{12} k(k-1)(k-2)(3 k-13) x^{3}\right) \sum_{n \geqslant k} S_{n, k} x^{n} \\
& +x Q_{k-1}^{\prime}(x)
\end{aligned}
$$

with $Q_{0}^{\prime}(x)=0, Q_{1}^{\prime}(x)=\frac{2(2-x)}{(1-x)^{2}}$ and $Q_{2}^{\prime}(x)=\frac{x^{2}\left(7-16 x+9 x^{2}-2 x^{3}\right)}{(1-2 x)^{2}(1-x)^{2}}$. Note that $H_{k}(x)=$ $\frac{x^{k}}{\prod_{j=1}^{k}(1-j x)}=\sum_{n \geqslant 0} S_{n, k} x^{n}$ and $\frac{1}{k !}\left(e^{x}-1\right)^{k}=\sum_{n \geqslant 0} S_{n, k} \frac{x^{n}}{n !}$, where $S_{n, k}$ denotes the Stirling number of the second kind (for example, see [12]). Thus, the exponential generating function $R_{k}(x)$ satisfies,

$$
\begin{aligned}
R_{k}(x) & =k \int_{0}^{x} R_{k}(t) d t+\int_{0}^{x} R_{k-1}(t) d t+3 \frac{\left(e^{x}-1\right)^{k}}{k !} \\
& +\frac{1}{2} k(k-5) \int_{0}^{x} \frac{\left(e^{t}-1\right)^{k}}{k !} d t-\left(k^{2}-4 k+2\right) \int_{0}^{x} \int_{0}^{t} \frac{\left(e^{r}-1\right)^{k}}{k !} d r d t \\
& -\frac{1}{12} k(k-1)(k-2)(3 k-13) \int_{0}^{x} \int_{0}^{t} \int_{0}^{r} \frac{\left(e^{s}-1\right)^{k}}{k !} d s d r d t,
\end{aligned}
$$

which is equivalent to

$$
\begin{aligned}
\frac{d^{3}}{d x^{3}} R_{k}(x) & =k \frac{d^{2}}{d x^{2}} R_{k}(x)+\frac{d^{2}}{d x^{2}} R_{k-1}(x)+3 \frac{d^{3}}{d x^{3}} \frac{\left(e^{x}-1\right)^{k}}{k !} \\
& +\frac{1}{2} k(k-5) \frac{d^{2}}{d x^{2}} \frac{\left(e^{x}-1\right)^{k}}{k !}-\left(k^{2}-4 k+2\right) \frac{d}{d x} \frac{\left(e^{x}-1\right)^{k}}{k !} \\
& -\frac{1}{12} k(k-1)(k-2)(3 k-13) \frac{\left(e^{x}-1\right)^{k}}{k !}
\end{aligned}
$$

with $R_{0}(x)=0, R_{1}(x)=2 x e^{x}+2 e^{x}-2$ and $R_{2}(x)=\frac{3}{4}-\frac{x}{2}-2(1+x) e^{x}+\left(2 x+\frac{5}{4}\right) e^{2 x}$. 
Define $R(x, y)=\sum_{k \geqslant 0} R_{k}(x) y^{k}$. Multiplying by $y^{k}$ and summing over $k \geqslant 3$ gives

$$
\begin{aligned}
& \frac{\partial^{3}}{\partial x^{3}} R(x, y)-y \frac{\partial^{2}}{\partial x^{2}} R(x, y)-y \frac{\partial^{3}}{\partial x^{2} y} R(x, y) \\
& =\frac{y\left(-y^{2}(3 y+4)+\left(12 y^{3}+6 y^{2}+24\right) e^{x}-12 y\left(y^{2}-6\right) e^{2 x}+34 y^{2} e^{3 x}+3 y^{3} e^{4 x}\right) e^{y\left(e^{x}-1\right)}}{12}
\end{aligned}
$$

By solving for $\frac{\partial^{2}}{\partial x^{2}} R(x, y)$, we obtain the following result.

Theorem 6. The exponential generating function $R(x, y)$ for the total of the site-perimeter over all set partitions of $[n]$ with exactly $k$ blocks satisfies

$$
\begin{aligned}
\frac{\partial^{2}}{\partial x^{2}} R(x, y)=\frac{y}{144} & e^{y\left(e^{x}-1\right)}\left(y^{2}(9 y+16)-12\left(4 y^{3}+3 y^{2}-24 x-72\right) e^{x}\right. \\
& \left.+72 y\left(y^{2}+12 x+14\right) e^{2 x}+4 y^{2}(102 x+5) e^{3 x}+3 y^{3}(12 x-11) e^{4 x}\right) .
\end{aligned}
$$

By Theorem 6, we have

$$
\begin{aligned}
\frac{\partial^{2}}{\partial x^{2}} R(x, y) & =\left(\frac{x}{4}-\frac{11}{48}\right) \frac{\partial^{4}}{\partial x^{4}} e^{y\left(e^{x}-1\right)}+\left(\frac{4 x}{3}+\frac{109}{72}\right) \frac{\partial^{3}}{\partial x^{3}} e^{y\left(e^{x}-1\right)} \\
& +\left(\frac{y^{2}}{2}+\frac{x}{4}+\frac{65}{16}\right) \frac{\partial^{2}}{\partial x^{2}} e^{y\left(e^{x}-1\right)}+\left(-\frac{y^{3}}{3}-\frac{3 y^{2}}{4}+\frac{x}{6}+\frac{47}{72}\right) \frac{\partial}{\partial x} e^{y\left(e^{x}-1\right)} \\
& +\left(\frac{y^{4}}{16}+\frac{y^{3}}{9}\right) e^{y\left(e^{x}-1\right)} .
\end{aligned}
$$

By comparing the coefficient of $x^{n} y^{k} / n$ ! in both sides, we get the following formula.

Corollary 7. The total of the site-perimeter over all set partitions of $[n+2]$ with exactly $k$ blocks is given by

$$
\begin{aligned}
\left(\frac{n}{4}+\frac{109}{72}\right) S_{n+3, k}-\frac{11}{48} S_{n+4, k} & +\left(\frac{4 n}{3}+\frac{65}{16}\right) S_{n+2, k}+\frac{1}{2} S_{n+2, k-2}+\left(\frac{n}{4}+\frac{47}{72}\right) S_{n+1, k} \\
& -\frac{1}{3} S_{n+1, k-3}-\frac{3}{4} S_{n+1, k-2}+\frac{n}{6} S_{n, k}+\frac{1}{16} S_{n, k-4}+\frac{1}{9} S_{n, k-3},
\end{aligned}
$$

and the total of the site-perimeter over all set partitions of $[n+2]$ is given by

$$
\left(\frac{n}{4}+\frac{109}{72}\right) B_{n+3}-\frac{11}{48} B_{n+4}+\left(\frac{4 n}{3}+\frac{73}{16}\right) B_{n+2}+\left(\frac{n}{4}-\frac{31}{72}\right) B_{n+1}+\left(\frac{n}{6}+\frac{25}{144}\right) B_{n}
$$

where $S_{n, k}$ denotes the Stirling number of the second kind and $B_{n}$ denotes the $n$th Bell number. 
Remark 8. Based on this work, we realized that there is a typo in the statement of Corollary 2.6 in [13]. More precisely, by (1) we have that, asymptotically, the total number of interior vertices (a vertex in $B$ is called an interior vertex if it is adjacent to exactly four different cells of bargraph $B)$ in set partitions of $[n+1]$ is given by

$$
\frac{n^{2} B_{n+1}}{3(\log (n)-\log \log n)}\left(1-\frac{1}{3(\log n-\log \log n)}\right)\left(1+O\left(\frac{\log n}{n}\right)\right) .
$$

We end the paper by emphasizing that all the above results have been compared with exact enumerations.

\section{Acknowledgements}

The author would like to thank the anonymous referee for pointing out a mistake in the previous version of (6), which changed the results in the third section.

\section{References}

[1] A. Blecher, C. Brennan, and A. Knopfmacher. Levels in bargraphs. Ars Math. Contemp. 9:287-300, 2015.

[2] A. Blecher, C. Brennan, and A. Knopfmacher. Peaks in bargraphs. Trans. Royal Soc. S. Afr. 71:97-103, 2016.

[3] A. Blecher, C. Brennan, and A. Knopfmacher. Combinatorial parameters in bargraphs. Quaest. Math. 39:619-635, 2016.

[4] A. Blecher, C. Brennan, and A. Knopfmacher. Walls in bargraphs. Online J. Analytic Combin. 12:619-635, 2017.

[5] A. Blecher, C. Brennan, A. Knopfmacher, and T. Mansour. The perimeter of words. Discr. Math. 340(10):2456-2465, 2017.

[6] A. Blecher, C. Brennan, A. Knopfmacher, and T. Mansour. The site-perimeter of words. Transactions Combin. 6(2):37-48, 2017.

[7] M. Bousquet-Mélou, and R. Brak. Exactly solved models of polyominoes and polygons. Chapter 3 of Polygons, Polyominoes and Polycubes, Lecture notes in physics, volume 775, 43-78, Springer, Berlin, Heidelberg 2009.

[8] E. R. Canfield. Engel's inequality for Bell numbers. J. Combin. Theory Ser. A 72:184-187, 1995.

[9] M. Delest, D. Gouyou-Beauchamps, and B. Vauquelin. Enumeration of parallelogram polyominoes with given bond and site perimeter. Graphs and Combin. 3:325-339, 1987.

[10] S. Feretić. A perimeter enumeration of column-convex polyominoes, Discrete Math. Theor. Comput. Sci. 9:57-84, 2007.

[11] P. A. MacMahon. Combinatory Analysis. Vol. I, II, Chelsea Publishing (American Mathematical Society), 2001. 
[12] T. Mansour. Combinatorics of Set Partitions. Chapman \& Hall/CRC, BocaRaton, FL, 2012.

[13] T. Mansour. Interior vertices in set partitions. Adv. Appl. Math. 101:60-69, 2018.

[14] T. Mansour, and A. Sh. Shabani. Interior vertices and edges in bargraphs. Notes on Number Theory and Discrete Mathematics, to appear.

[15] T. Mansour, and M. Shattuck. Combinatorial parameters on bargraphs of permutations. Trans. Combin. 7(2):1-16, 2018.

[16] T. Mansour, and M. Shattuck. Bargraph statistics on words and set partitions. J. Diff. Equat. Appl. 23(6):1025-1046, 2017.

[17] T. Prellberg, and R. Brak. Critical exponents from nonlinear functional equations for partially directed cluster models. J. Stat. Phys. 78:701-730, 1995.

[18] M. Shattuck. Recounting the number of rises, levels, and descents in finite set partitions. Integers 10:179-185, 2010.

[19] D. Stanton, and D. White. Constructive Combinatorics. Springer, New York, 1986. 